\title{
STUDY ON DEBRIS FLOW FORECASTING USING A RAINFALL TRIGGERING INDEX
}

\author{
DONG HO NAM, DONG HO KANG, SUK HO LEE \& BYUNG SIK KIM \\ Department of Urban and Environmental Disaster Prevention Engineering, \\ Kangwon National University, Republic of Korea
}

\begin{abstract}
At present, there has been a wide range of studies on debris flow in Korea, more specifically, on rainfall characteristics that trigger debris flow including rainfall intensity, rainfall duration, and preceding rainfall. The forecasting of debris flow relies on the criteria for debris flow forecasting and warning by the Korea Forest Service. Despite this, it has been found that most incidents of debris flow were caused by rainfall above the level of debris flow watch, maximum hourly rainfall, extensive damage was caused even under the watch level. under these circumstances, we estimated a rainfall triggering index (RTI) using the main factors that trigger debris flow rainfall, rainfall intensity, and cumulative rainfall to design a more sophisticated watch/warning criteria than those by the KFS. The RTI was classified into attention, caution, and warning, and was assessed through the application of two debris flow incidents that occurred in Umyeon Mountain, Seoul, and Cheongju, Inje, causing serious damage and casualties. Moreover, we reviewed the feasibility of the RTI by comparing it with the KFS's debris flow forecasting and warning criteria.

Keywords: debris flow watch/warning criteria, rainfall intensity, preceding rainfall, debris flow forecasting and warning, rainfall triggering index (RTI).
\end{abstract}

\section{INTRODUCTION}

The recent extreme weather events across the globe due to climate change have become serious challenges. Over the past 100 years, abnormal climate conditions, such as rising temperature, increasing precipitation, and increasing rainfall days, have been observed in Korea [1]. As a result, there have been more occurrences of natural disasters such as heavy rain, storms, droughts, and heavy snow. In particular, typhoons and heavy rainfall in summer, from June to September, are more likely to cause secondary debris flow damage [2]. A debris flow is a complex natural disaster triggered by the combination of a flood and a landslide. Mainly due to heavy rainfall, debris flows cause increased flow rate, soil loss, and large-scale rock movement, which could result in massive damage to human lives and property [3]. Recently, Korea experienced many cases of debris flow damage nationwide: Inje-gun and Pyeongchang-gun, Gangwon-do in 2006; Seoul, Chuncheon-si, and Pochen in 2011; Samcheok in 2012; Busan in 2014; and Cheongju-si in 2017. Analysis of rainfall characteristics that could cause debris flows is very important for the establishment of a debris flow early warning system and the development and maintenance of a disaster prevention system to minimize debris flow damage. However, there have been few studies on debris flow forecasting and warning using rainfall characteristics analysis [4].

Therefore, to predict debris flows that could be caused by rainfall, this study focused on the estimation of the rainfall triggering index (RTI) using the estimation method developed Jan and Lee [5]. To this end, rainfall intensity and accumulated rainfall data of the 50 spots that experienced damage by debris flows from 2012 to 2013, taking into consideration preceding data (for 24 hours) were thus used. The estimated RTI was divided into three levels: advisory (10\%), warning (70\%), and emergency warning (over 90\%). These were subsequently applied to previous debris flows that caused serious damage in Umyeonsan Mountain, Seoul, Cheongju-si, and Inje-gun to identify the maximum prevention or response 
period by estimating the RTI levels at 3, 6, and 12 hours before the debris flows occurred. The feasibility of the RTI based on the debris flow caution and warning criteria provided by the Korea Forest Service (KFS) [6] was also reviewed.

\section{STUDY METHODS}

In Korea, the debris flow forecast criteria are defined by the KFS (Table 1), but criteria for debris flow forecasting have yet to be clearly established. Since the debris flows that happened in Umyeonsan Mountain in Seoul and Majeoksan Mountain in Chuncheon caused massive loss of human lives and property, some domestic research institutes, including the KFS, the Korea Institute of Geoscience and Mineral Resources (KIGAM), the Seoul Institute (SI), and the Korea Expressway Corporation (KEC), have developed their own debris flow assessment criteria and conducted independent assessments on debris flow-prone areas [7].

Therefore, to suggest more specific and detailed forecasting and warning criteria for debris flows, rainfall data of 50 spots in Gangwon-do that were hit by debris flows from 2012 to 2013 were collected (Fig. 1). When the rainfall lasted for at least 24 consecutive hours within the total rainfall period of up to 7 days right before the debris flow occurred, a direct factor that could trigger the event, it was considered preceding rainfall. However, when it lasted for less than 24 consecutive hours, it was excluded from the RTI estimation. The RTI estimated for the 50 spots were classified into the three levels described above: "advisory" with a debris flow risk of $10-70 \%$, "warning" with a risk of $70-90 \%$, and "emergency warning” with a risk of over $90 \%$. In addition, for comparison with the debris flow forecasting criteria by the KFS, the RTI classification was also applied to the cases of Umyeonsan Mountain in 2011, Cheongju-si, Chungbuk in 2017, and Inje-gun in 2006 to identify the maximum prevention or response period, if it had been forecasted by estimating the RTI 3, 6, and 12 hours before the debris flows.

Table 1: Debris flow forecasting standard (Korea forest service).

\begin{tabular}{|l|c|c|c|}
\hline & $\begin{array}{c}\text { Maximum hourly } \\
\text { rainfall }(\mathrm{mm})\end{array}$ & $\begin{array}{c}\text { Daily rainfall } \\
(\mathrm{mm})\end{array}$ & $\begin{array}{c}\text { Continuous } \\
\text { rainfall (mm) }\end{array}$ \\
\hline Debris flow warning & $20-30$ & $80-150$ & $100-200$ \\
\hline Debris flow alarm & $>30$ & $>150$ & $>200$ \\
\hline
\end{tabular}

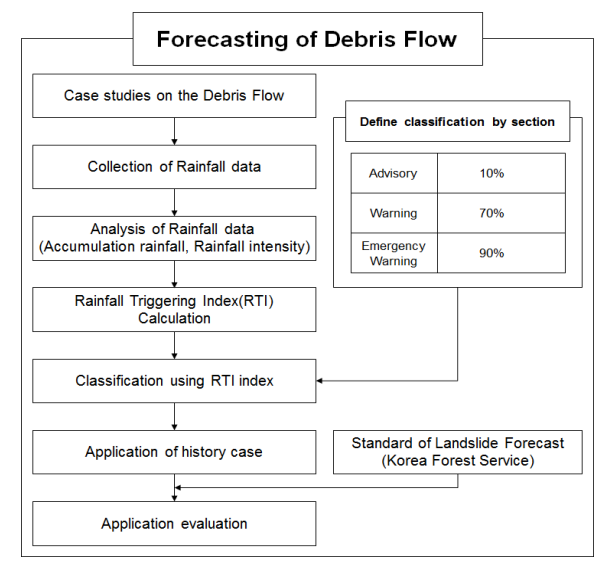

Figure 1: Flowchart for debris flow forecasting. 


\section{THEORETICAL BACKGROUND}

\subsection{Rainfall triggering index (RTI) model}

The RTI model developed by Jan and Lee [5] makes real-time forecasting of debris flow caused by rainfall, using rainfall intensity (I) and accumulated rainfall $\left(R_{t}\right)$. The RTI is estimated by the following eqn (1).

$$
\mathrm{RTI}=\mathrm{I} \times R_{t},
$$

where (I) is rainfall intensity for an hour $(\mathrm{mm} / \mathrm{hr})$, and $\left(R_{t}\right)$ is accumulated rainfall right before the debris flow began. In addition, for $\left(R_{t}\right)$, when the rainfall lasted for at least 24 consecutive hours within the total rainfall period of up to 7 days right before the debris flow a direct factor that could trigger the event it was considered preceding rainfall [8]. However, when it lasted for less than 24 consecutive hours, it was excluded from the estimation.

The occurrence of debris flows is directly affected by rainfall and particularly influenced by accumulated rainfall and rainfall intensity. The existing debris flow forecasting and warning system is based on accumulated rainfall, rainfall intensity, or daily rainfall. However, RTI uses both accumulated rainfall and rainfall intensity to predict debris flows. Fig. 2 illustrates the concept of the RTI.

\section{RESULTS AND DISCUSSIONS}

\subsection{RTI estimation}

The rainfall intensity and accumulated rainfall of 50 spots in Gangwon-do hit by debris flows from 2012 to 2013 were estimated, taking into consideration preceding rainfall. The RTI for each spot was subsequently estimated. In addition, the RTI estimated for the 50 spots above, the risk of debris flows was classified into three levels: $10-70 \%, 70-90 \%$, and over $90 \%$. It was found that when the risk is $10 \%$, the RTI was $664 ; 70 \%$ for 1430 ; and over $90 \%$ for 1751 (Fig. 4). Fig. 3 illustrates the locations of debris flows and rainfall stations, while Table 2 shows damage occurrence time, duration of rainfall, accumulated rainfall, rainfall intensity, and RTI.

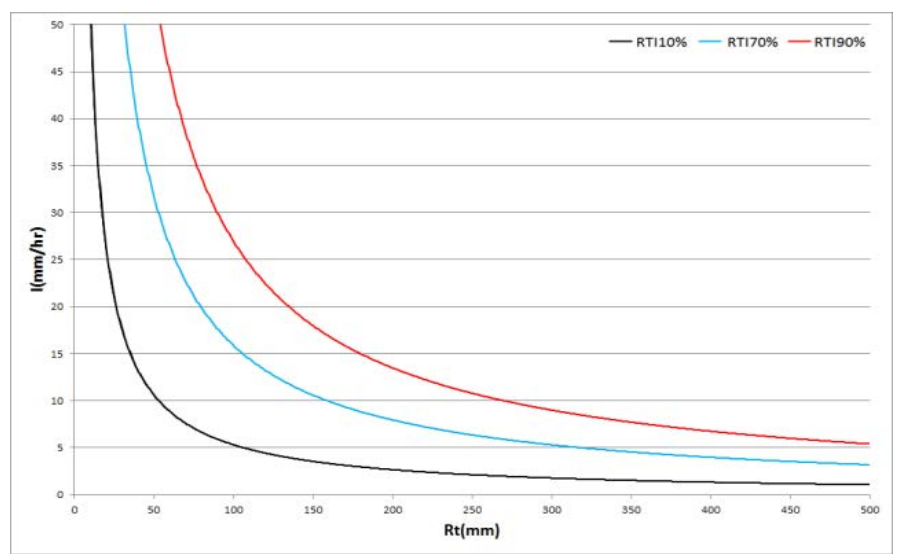

Figure 2: Concept of rainfall triggering index. 


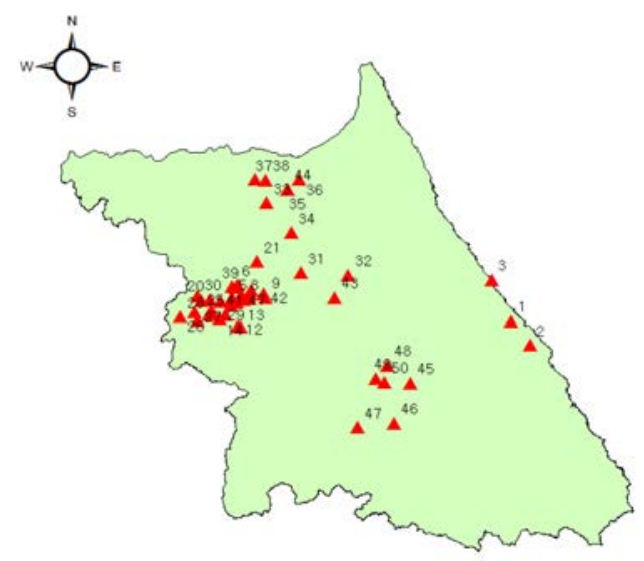

A Occurrence point of landslide and debris flow

Figure 3: Occurrence status debris flow.

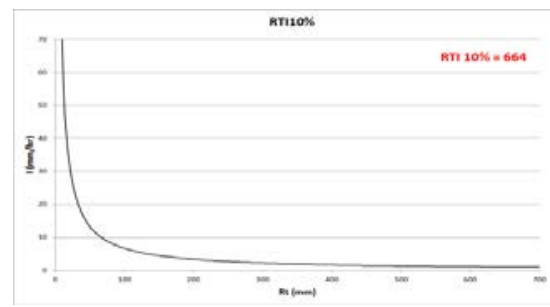

(a) RTI $10 \%$

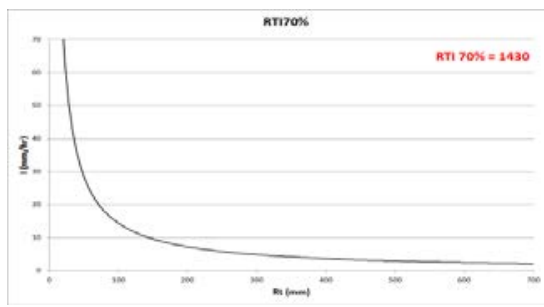

(b) RTI 70\%

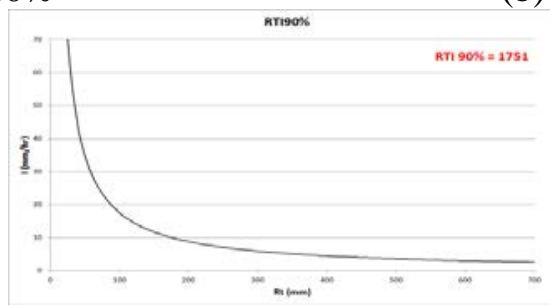

(c) RTI 90\%

Figure 4: Estimation of rainfall triggering index.

For forecasting and warning of a debris flow occurrence, there is a need to establish relevant criteria. Using the previously estimated RTI, it was classified as 10\% advisory, $70 \%$ warning, and over $90 \%$ emergency warning level. Also, most of the RTI points of the 50 spots were located under the $70 \%$ risk line (Fig. 5).

The points under the $10 \%$ line were taken into consideration due to the geological and geographical properties of the Gangwon-do region, which have diverse compositions with a vast geological time scale from the Precambrian Era to the Quaternary Period. Among the varied compositions of the area are the Kyonggi Massif, Phyeongnam Basin, Ongjin Basin, 
Table 2: Estimation of rainfall triggering index.

\begin{tabular}{|c|c|c|c|c|c|c|}
\hline No & Occurrence area & $\begin{array}{c}\text { Occurrence } \\
\text { time }\end{array}$ & $\begin{array}{l}\text { Duration of } \\
\text { rainfall (hr) }\end{array}$ & $\begin{array}{c}\text { Accumulation } \\
\text { rainfall }(\mathrm{mm})\end{array}$ & $\begin{array}{l}\text { Intensity } \\
(\mathrm{mm} / \mathrm{hr})\end{array}$ & RTI \\
\hline 1 & $\begin{array}{l}\text { 438, Imgok-ri, Gangdong- } \\
\text { myeon, Gangneung-si, } \\
\text { Gangwon-do }\end{array}$ & $\begin{array}{l}\text { 2012-09-17 } \\
11: 05\end{array}$ & 30 & 149 & 4.98 & 745 \\
\hline 2 & $\begin{array}{l}\text { 143, Gyo-dong, } \\
\text { Gangneung-si Gangwon-do }\end{array}$ & $\begin{array}{l}2012-09-17 \\
17: 24\end{array}$ & 36 & 235 & 6.52 & 1534 \\
\hline 3 & $\begin{array}{l}\text { 1-3, Sodolsaet-gil, } \\
\text { Jumunjin-eup, Gangneung- } \\
\text { si, Gangwon-do }\end{array}$ & $\begin{array}{l}\text { 2012-09-17 } \\
10: 00\end{array}$ & 26 & 88 & 3.38 & 297 \\
\hline 4 & $\begin{array}{l}\text { 39, Dochon-ri, Nam- } \\
\text { myeon, Yanggu-gun, } \\
\text { Gangwon-do }\end{array}$ & $\begin{array}{l}\text { 2013-07-18 } \\
20: 30\end{array}$ & 10 & 31 & 3 & 93 \\
\hline 5 & $\begin{array}{l}\text { 1, Deokgok-ri, Dong- } \\
\text { myeon, Yanggu-gun, } \\
\text { Gangwon-do }\end{array}$ & $\begin{array}{l}\text { 2013-07-18 } \\
20: 30\end{array}$ & 8 & 168 & 21 & 3528 \\
\hline 6 & $\begin{array}{l}\text { 1-2, Paldang-ri, Dong- } \\
\text { myeon, Yanggu-gun, } \\
\text { Gangwon-do }\end{array}$ & $\begin{array}{l}\text { 2013-07-18 } \\
20: 30\end{array}$ & 8 & 168 & 21 & 3528 \\
\hline 7 & $\begin{array}{l}\text { 3, Gobangsan-ri, Bangsan- } \\
\text { myeon, Yanggu-gun, } \\
\text { Gangwon-do }\end{array}$ & $\begin{array}{l}\text { 2013-07-18 } \\
20: 30\end{array}$ & 8 & 168 & 21 & 3528 \\
\hline 8 & $\begin{array}{l}\text { 4, Gobangsan, Bangsan- } \\
\text { myeon, Yanggu-gun, } \\
\text { Gangwon-do }\end{array}$ & $\begin{array}{l}\text { 2013-07-18 } \\
20: 30\end{array}$ & 8 & 168 & 21 & 3528 \\
\hline 9 & $\begin{array}{l}\text { 42, Cheonmi-ri, Bangsan- } \\
\text { myeon, Yanggu-gun, } \\
\text { Gangwon-do }\end{array}$ & $\begin{array}{l}2013-07-18 \\
20: 30\end{array}$ & 8 & 168 & 21 & 3528 \\
\hline 10 & $\begin{array}{l}\text { 1-22, Sangmuryong-ri, } \\
\text { Yanggu-eup, Yanggu-gun, } \\
\text { Gangwon-do }\end{array}$ & $\begin{array}{l}\text { 2013-07-18 } \\
20: 30\end{array}$ & 9 & 125 & 13.88 & 1736 \\
\hline 11 & $\begin{array}{l}\text { 5, Eoron-ri, Nam-myeon, } \\
\text { Inje-gun, Gangwon-do }\end{array}$ & $\begin{array}{l}\text { 2013-07-14 } \\
08: 00 \\
\end{array}$ & 96 & 79 & 9.87 & 1290 \\
\hline 12 & $\begin{array}{l}\text { Gangwon Province, Sanam- } \\
\text { ri, Sangnam-myeon, } \\
\text { Yanggu-gun, Gangwon-do }\end{array}$ & $\begin{array}{l}\text { 2013-07-14 } \\
\text { 08:00 }\end{array}$ & 72 & 212 & 2.94 & 623 \\
\hline 13 & $\begin{array}{l}\text { 97, Baegyang-ri, Namsan- } \\
\text { myeon, Chuncheon-si, } \\
\text { Gangwon-do }\end{array}$ & $\begin{array}{l}\text { 2013-07-15 } \\
00: 00\end{array}$ & 75 & 281 & 3.74 & 1052 \\
\hline 14 & $\begin{array}{l}\text { 26, Changchon-ri, Namsan- } \\
\text { myeon, Chuncheon-si, } \\
\text { Gangwon-do }\end{array}$ & $\begin{array}{l}\text { 2013-07-15 } \\
00: 00\end{array}$ & 120 & 333 & 3.7 & 1092 \\
\hline 15 & $\begin{array}{l}\text { 1-2, Goeun-ri, Dongnae- } \\
\text { myeon, Chuncheon-si, } \\
\text { Gangwon-do }\end{array}$ & $\begin{array}{l}\text { 2013-07-15 } \\
00: 00\end{array}$ & 168 & 477 & 2.84 & 1354 \\
\hline 16 & $\begin{array}{l}\text { 70, Goeun-ri, Dongnae- } \\
\text { myeon, Chuncheon-si, } \\
\text { Gangwon-do }\end{array}$ & $\begin{array}{l}\text { 2013-07-15 } \\
00: 00\end{array}$ & 168 & 477 & 2.84 & 1354 \\
\hline 17 & $\begin{array}{l}\text { 1, Saam-ri, Dongnae- } \\
\text { myeon, Chuncheon-si, } \\
\text { Gangwon-do }\end{array}$ & $\begin{array}{l}\text { 2013-07-15 } \\
00: 00\end{array}$ & 168 & 477 & 2.84 & 1354 \\
\hline
\end{tabular}


Table 2: Continued.

\begin{tabular}{|c|c|c|c|c|c|c|}
\hline No & Occurrence area & $\begin{array}{l}\text { Occurrence } \\
\text { time }\end{array}$ & $\begin{array}{l}\text { Duration of } \\
\text { rainfall }(\mathrm{hr})\end{array}$ & $\begin{array}{l}\text { Accumulation } \\
\text { rainfall (mm) }\end{array}$ & $\begin{array}{l}\text { Intensity } \\
(\mathrm{mm} / \mathrm{hr})\end{array}$ & RTI \\
\hline 18 & $\begin{array}{l}\text { 32, Saam-ri, Dongnae- } \\
\text { myeon, Chuncheon-si, } \\
\text { Gangwon-do }\end{array}$ & $\begin{array}{l}\text { 2013-07-15 } \\
00: 00\end{array}$ & 168 & 477 & 2.84 & 1354 \\
\hline 19 & $\begin{array}{l}\text { 2, Sinchon-ri, Dongnae- } \\
\text { myeon, Chuncheon-si, } \\
\text { Gangwon-do }\end{array}$ & $\begin{array}{l}\text { 2013-07-15 } \\
00: 00\end{array}$ & 168 & 477 & 2.84 & 1354 \\
\hline 20 & $\begin{array}{l}\text { 84-3, Gamjeong-ri, Dong- } \\
\text { myeon, Chuncheon-si, } \\
\text { Gangwon-do }\end{array}$ & $\begin{array}{l}\text { 2013-07-14 } \\
00: 00\end{array}$ & 64 & 154 & 2.4 & 370 \\
\hline 21 & $\begin{array}{l}\text { 38-1, Sanggeol-ri, Dong- } \\
\text { myeon, Chuncheon-si, } \\
\text { Gangwon-do }\end{array}$ & $\begin{array}{l}\text { 2013-07-14 } \\
\text { 09:08 }\end{array}$ & 74 & 343 & 4.63 & 1589 \\
\hline 22 & $\begin{array}{l}\text { 82, Sanggeol-ri, Dong- } \\
\text { myeon, Chuncheon-si, } \\
\text { Gangwon-do }\end{array}$ & $\begin{array}{l}\text { 2013-07-14 } \\
\text { 09:08 }\end{array}$ & 74 & 343 & 4.63 & 1589 \\
\hline 23 & $\begin{array}{l}\text { 53, Sanjeong-ri, Sanggeol- } \\
\text { myeon, Chuncheon-si, } \\
\text { Gangwon-do }\end{array}$ & $\begin{array}{l}\text { 2013-07-14 } \\
\text { 09:08 }\end{array}$ & 74 & 343 & 4.63 & 1589 \\
\hline 24 & $\begin{array}{l}\text { 353, Sanjeong-ri, Sanggeol- } \\
\text { myeon, Chuncheon-si, } \\
\text { Gangwon-do }\end{array}$ & $\begin{array}{l}\text { 2013-07-14 } \\
\text { 09:08 }\end{array}$ & 74 & 343 & 4.63 & 1589 \\
\hline 25 & $\begin{array}{l}\text { 2-1, Sini-ri, Dong-myeon, } \\
\text { Chuncheon-si, Gangwon-do }\end{array}$ & $\begin{array}{l}\text { 2013-07-14 } \\
\text { 09:08 }\end{array}$ & 74 & 343 & 4.63 & 1589 \\
\hline 26 & $\begin{array}{l}\text { 1-1(2), Samgyeol-ri, Dong- } \\
\text { myeon, Chuncheon-si, } \\
\text { Gangwon-do }\end{array}$ & $\begin{array}{l}\text { 2013-07-14 } \\
\text { 09:08 }\end{array}$ & 74 & 343 & 4.63 & 1589 \\
\hline 27 & $\begin{array}{l}\text { 1-1(11), Samgyeol-ri, } \\
\text { Dong-myeon, Chuncheon- } \\
\text { si, Gangwon-do }\end{array}$ & $\begin{array}{l}\text { 2013-07-14 } \\
\text { 09:08 }\end{array}$ & 74 & 343 & 4.63 & 1589 \\
\hline 28 & $\begin{array}{l}\text { 61-32, Ottan-ri, Sabuk- } \\
\text { myeon, Chuncheon-si, } \\
\text { Gangwon-do }\end{array}$ & $\begin{array}{l}\text { 2013-07-15 } \\
00: 00\end{array}$ & 151 & 61 & 8.71 & 434 \\
\hline 29 & $\begin{array}{l}\text { 115-1, Wonpyeong-ri, } \\
\text { Sabuk-myeon, Chuncheon- } \\
\text { si, Gangwon-do }\end{array}$ & $\begin{array}{l}\text { 2013-07-14 } \\
\text { 09:08 }\end{array}$ & 104 & 222 & 2.96 & 591 \\
\hline 30 & $\begin{array}{l}\text { 1-1, Samgyeol-ri, Dong- } \\
\text { myeon, Chuncheon-si, } \\
\text { Gangwon-do }\end{array}$ & $\begin{array}{l}\text { 2013-07-14 } \\
\text { 09:08 }\end{array}$ & 74 & 343 & 4.63 & 1589 \\
\hline 31 & $\begin{array}{l}\text { 27, Bongmyeong-ri, } \\
\text { Dongsan-myeon, } \\
\text { Chuncheon-si, Gangwon-do }\end{array}$ & $\begin{array}{l}\text { 2013-07-14 } \\
\text { 09:08 }\end{array}$ & 74 & 343 & 4.63 & 1589 \\
\hline 32 & $\begin{array}{l}\text { 31, Bongmyeong-ri, } \\
\text { Dongsan-myeon, } \\
\text { Chuncheon-si, Gangwon-do }\end{array}$ & $\begin{array}{l}\text { 2013-07-14 } \\
\text { 09:08 }\end{array}$ & 88 & 359 & 4.08 & 1464 \\
\hline 33 & $\begin{array}{l}\text { 27-1, Bongmyeong-ri, } \\
\text { Dongsan-myeon, } \\
\text { Chuncheon-si, Gangwon-do }\end{array}$ & $\begin{array}{l}\text { 2013-07-15 } \\
00: 00\end{array}$ & 88 & 359 & 4.08 & 1464 \\
\hline 34 & $\begin{array}{l}\text { 191, Wonchang-ri, } \\
\text { Dongsan-myeon, } \\
\text { Chuncheon-si, Gangwon-do }\end{array}$ & $\begin{array}{l}\text { 2013-07-15 } \\
00: 00\end{array}$ & 88 & 359 & 4.08 & 1464 \\
\hline
\end{tabular}


Table 2: Continued.

\begin{tabular}{|c|c|c|c|c|c|c|}
\hline No & Occurrence area & $\begin{array}{l}\text { Occurrence } \\
\text { time }\end{array}$ & $\begin{array}{l}\text { Duration of } \\
\text { rainfall (hr) }\end{array}$ & $\begin{array}{l}\text { Accumulation } \\
\text { rainfall (mm) }\end{array}$ & $\begin{array}{r}\text { Intensity } \\
(\mathrm{mm} / \mathrm{hr})\end{array}$ & RTI \\
\hline 35 & $\begin{array}{l}\text { 27-1, Joyang-ri, Dongsan- } \\
\text { myeon, Chuncheon-si, } \\
\text { Gangwon-do }\end{array}$ & $\begin{array}{l}\text { 2013-07-15 } \\
00: 00\end{array}$ & 88 & 359 & 4.08 & 1464 \\
\hline 36 & $\begin{array}{l}\text { 5, Jiam-ri, Sabuk-myeon, } \\
\text { Chuncheon-si, Gangwon-do }\end{array}$ & $\begin{array}{c}\text { 2013-07-15 } \\
00: 00\end{array}$ & 168 & 310 & 3.37 & 1226 \\
\hline 37 & $\begin{array}{l}\text { 59, Dangnim-ri, Seo-myeon, } \\
\text { Chuncheon-si, Gangwon-do }\end{array}$ & $\begin{array}{c}\text { 2013-07-15 } \\
00: 00 \\
\end{array}$ & 85 & 179 & 8.14 & 1145 \\
\hline 38 & $\begin{array}{l}\text { 109, Deokduwon-ri, } \\
\text { Deokduwon-myeon, } \\
\text { Chuncheon-si, Gangwon-do }\end{array}$ & $\begin{array}{l}\text { 2013-07-15 } \\
00: 00\end{array}$ & 76 & 289 & 3.8 & 1098 \\
\hline 39 & $\begin{array}{l}\text { 63, Seoksa-dong, } \\
\text { Chuncheon-si, Gangwon-do }\end{array}$ & $\begin{array}{c}2013-07-15 \\
00: 00\end{array}$ & 76 & 289 & 3.8 & 1098 \\
\hline 40 & $\begin{array}{l}\text { 94-17, Jeong-ri ,Sindong- } \\
\text { myeon, Chuncheon-si, } \\
\text { Gangwon-do }\end{array}$ & $\begin{array}{l}\text { 2013-07-15 } \\
\text { 00:00 }\end{array}$ & 76 & 289 & 3.8 & 1098 \\
\hline 41 & $\begin{array}{l}\text { 100-1, Jeong-ri ,Sindong- } \\
\text { myeon, Chuncheon-si, } \\
\text { Gangwon-do }\end{array}$ & $\begin{array}{l}\text { 2013-07-15 } \\
00: 00\end{array}$ & 76 & 289 & 3.8 & 1098 \\
\hline 42 & $\begin{array}{l}\text { 79-2, Palmi-ri, Sindong- } \\
\text { myeon, Chuncheon-si, } \\
\text { Gangwon-do }\end{array}$ & $\begin{array}{l}\text { 2013-07-15 } \\
00: 00\end{array}$ & 76 & 289 & 3.8 & 1098 \\
\hline 43 & $\begin{array}{l}\text { 1-1, Pumgeol-ri, Dong- } \\
\text { myeon, Chuncheon-si, } \\
\text { Gangwon-do }\end{array}$ & $\begin{array}{l}\text { 2013-07-15 } \\
00: 00\end{array}$ & 74 & 343 & 4.64 & 1589 \\
\hline 44 & $\begin{array}{l}\text { 614, Sin-ri, Daehwa-myeon, } \\
\text { Pyeongchang-gun, Gangwon- } \\
\text { do }\end{array}$ & $\begin{array}{l}\text { 2013-07-15 } \\
12: 00\end{array}$ & 64 & 333 & 5.2 & 1732 \\
\hline 45 & $\begin{array}{l}\text { 14, Bangnim-ri, Bangnim- } \\
\text { myeon, Pyeongchang-gun, } \\
\text { Gangwon-do }\end{array}$ & $\begin{array}{l}\text { 2013-07-15 } \\
12: 00\end{array}$ & 46 & 248 & 3.8 & 961 \\
\hline 46 & $\begin{array}{l}\text { 333-1, Chiksari-gil, } \\
\text { Bangnim-myeon, } \\
\text { Pyeongchang-gun, Gangwon- } \\
\text { do }\end{array}$ & $\begin{array}{l}\text { 2013-07-15 } \\
12: 00\end{array}$ & 59 & 209 & 3.54 & 740 \\
\hline 47 & $\begin{array}{l}\text { 56-1, Deokgeo-ri, } \\
\text { Bongpyeong-myeon, } \\
\text { Pyeongchang-gun, Gangwon- } \\
\text { do }\end{array}$ & $\begin{array}{l}\text { 2013-07-15 } \\
12: 00\end{array}$ & 66 & 422 & 6.4 & 2698 \\
\hline 48 & $\begin{array}{l}\text { 310, Pyeongchon-ri, } \\
\text { Bongpyeong-myeon, } \\
\text { Pyeongchang-gun, Gangwon- } \\
\text { do }\end{array}$ & $\begin{array}{l}\text { 2013-07-15 } \\
12: 00\end{array}$ & 66 & 422 & 6.4 & 2698 \\
\hline 49 & $\begin{array}{l}\text { 251-1, Baegokpo-ri, } \\
\text { Yongpyeong-myeon, } \\
\text { Pyeongchang-gun, Gangwon- } \\
\text { do }\end{array}$ & $\begin{array}{l}\text { 2013-07-15 } \\
12: 00\end{array}$ & 70 & 349 & 4.98 & 1740 \\
\hline 50 & $\begin{array}{l}\text { 166-5, Imokjeong-ri, } \\
\text { Yongpyeong-myeon, } \\
\text { Pyeongchang-gun, Gangwon- } \\
\text { do }\end{array}$ & $\begin{array}{l}\text { 2013-07-15 } \\
12: 00\end{array}$ & 39 & 128 & 3.28 & 420 \\
\hline
\end{tabular}




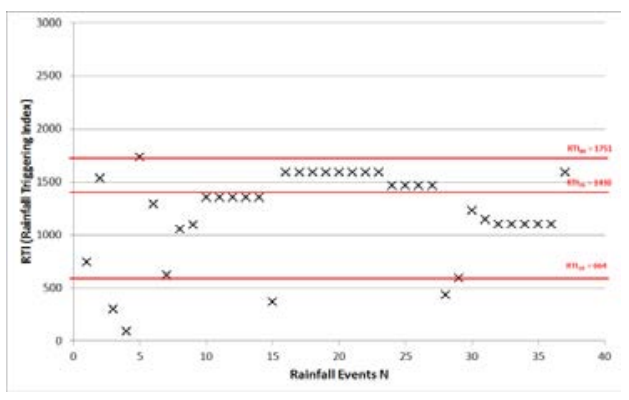

Figure 5: Establish of debris flow forecasting using RTI.

Yeongnam Basin, etc. The region's typical rocks include Jurassic granites (37\%), banded gneiss (33\%), and pelite (17\%) (Fig. 6). In general, metamorphic and granitic rocks are likely to cause more debris flows as their weathered residual soil could have small cohesion and internal friction angles, depending on the geological weathering conditions.

\subsection{Debris flow forecasting/warning and response time analysis}

For comparison with the debris flow forecasting criteria by the KFS, the RTI classification was also applied to the cases in Umyeonsan Mountain in 2011, Cheongju-si, Chungbuk in 2017, and Inje-gun in 2006 to identify the maximum response time if it had been forecasted by estimating the RTI 3, 6, and 12 hours before the debris flows. The results showed that for the case of Umyeonsan, the RTI was estimated as 1897 due to the 50-hour rainfall, which means that there was over $90 \%$ debris flow risk ("emergency warning") with less than $10 \%$ at 12 hours prior to the event, "advisory" at 6 hours prior, and "warning" at 3 hours prior. These results indicate that response could have been provided three hours before the debris flow occurred (Fig. 7). According to the KFS criteria, it was "caution" from 36 to 43 hours of daily rainfall and "warning" after 44 hours. Similarly, for accumulated rainfall, it was "caution" from 36 to 43 hours and "warning” after 44 hours. For rainfall intensity, the RTI did not fall into "caution" nor "warning” levels (Fig. 8). Table 3 shows the results of the RTIbased forecasting and KFS’s forecasting.

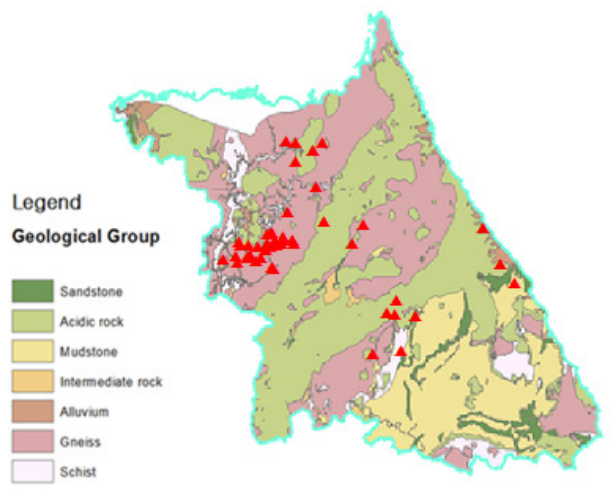

Figure 6: Modified geological map of Gangwon-do province. 


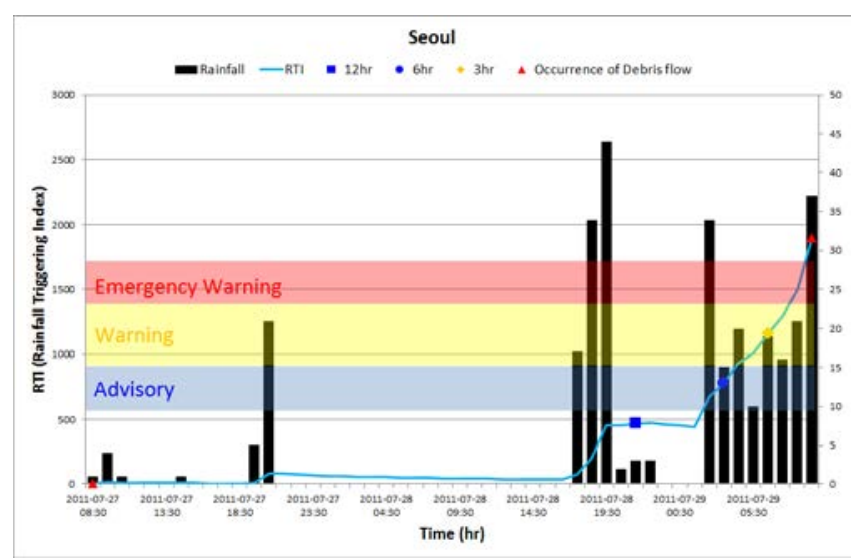

Figure 7: Debris flow forecasting using RTI (Seoul).

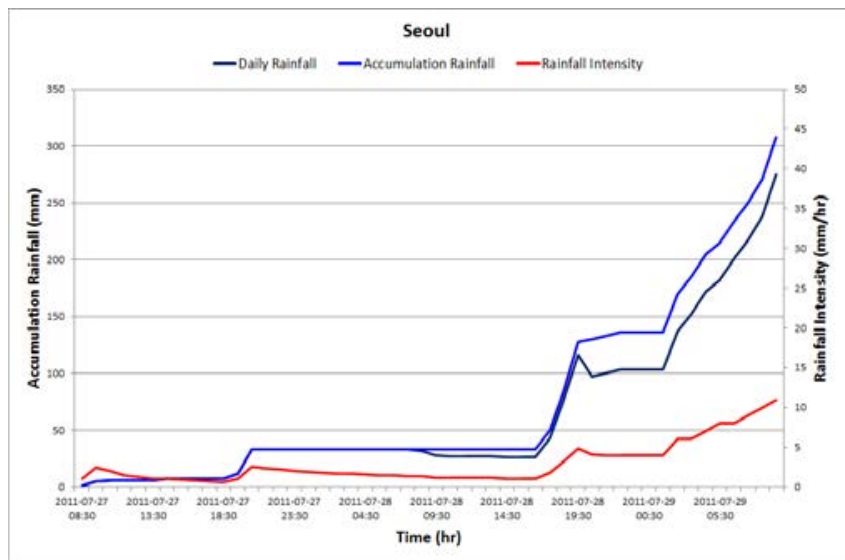

Figure 8: Debris flow forecasting using the Korea forest service forecasting criteria (Seoul).

Table 3: Estimation of rainfall triggering index (Seoul).

\begin{tabular}{|l|c|c|c|c|c|c|c|}
\hline $\begin{array}{l}\text { Occurrence } \\
\text { area }\end{array}$ & $\begin{array}{c}\text { Occurrence } \\
\text { time }\end{array}$ & $\begin{array}{c}\text { Duration } \\
\text { of rainfall } \\
\text { (hr) }\end{array}$ & $\begin{array}{c}\text { Accumulative } \\
\text { rainfall (mm) }\end{array}$ & $\begin{array}{c}\text { Intensity } \\
(\mathrm{mm} / \mathrm{hr})\end{array}$ & RTI & $\begin{array}{c}\text { RTI } \\
\text { forecasting }\end{array}$ & $\begin{array}{c}\text { Debris flow } \\
\text { forecasting }\end{array}$ \\
\hline $\begin{array}{c}2011-07-27 \\
08: 30\end{array}$ & 50 & 308 & 6.16 & 1897 & $\begin{array}{c}\text { Emergency } \\
\text { warning }\end{array}$ & Alarm \\
\cline { 2 - 8 } & 3 hours & 47 & 234 & 4.98 & 1165 & Warning & Alarm \\
\cline { 2 - 8 } \\
$\begin{array}{l}\text { Mountain, } \\
\text { Seoul }\end{array}$ & 6 hours & 44 & 185 & 4.20 & 777 & Advisory & Warning \\
\cline { 2 - 8 } & 12 hours & 38 & 133 & 3.5 & 466 & Advisory & Warning \\
\hline
\end{tabular}


For the case of Cheongju, the RTI was estimated at 2332. This means that there was over a $90 \%$ risk of debris flow ("emergency warning") with less than $10 \%$ at 12 , 6 , and even 3 hours before the event (Fig. 9). This means that the short, intense, almost five-hour rainfall caused the debris flow, thus making it difficult to estimate the response time before the disaster occurred. According to the KFS criteria, the daily rainfall was at the "caution" level for 1 hour at 39 hours and at the "warning" level from 40 to 42 hours. The accumulated rainfall was at the "caution" level for 2 hours from 39 to 40 hours and the "warning" level from 41 to 42 hours. The rainfall intensity did not fall into the "caution" or "warning" levels (Fig. 10). Table 4 shows the results of the RTI-based forecasting and KFS's forecasting for the case of Chungbuk.

For the case of Inje, despite the relatively short rainfall, the RTI was at 1855 . This means that there was over $90 \%$ of the debris flow risk with less than $10 \%$ at 12,6 , and even 3 hours before the occurrence (Fig. 11). As shown in the case of Cheongju, it can be assumed that a short but intense rainfall caused the disaster. According to the KFS criteria, both daily rainfall and rainfall intensity did not fall even into the "caution" level; only the accumulated rainfall was at the "caution" level one hour prior to the occurrence (Fig. 12). Table 5 shows the results of both methods for the case of Inje.

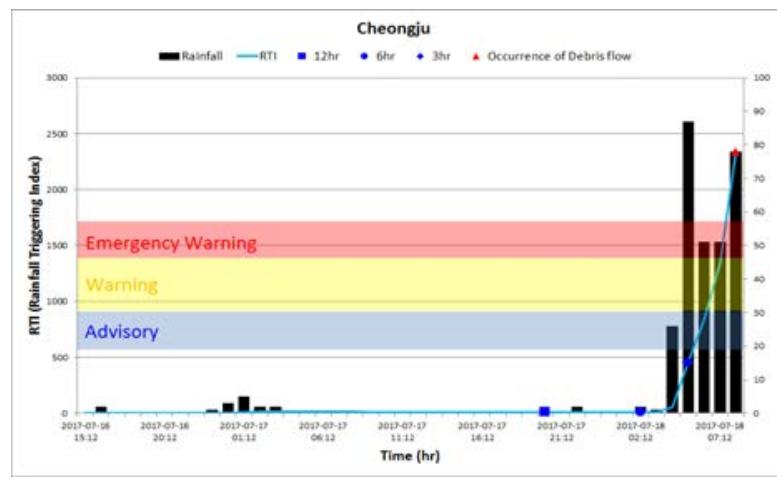

Figure 9: Debris flow forecasting using RTI (Cheongju).

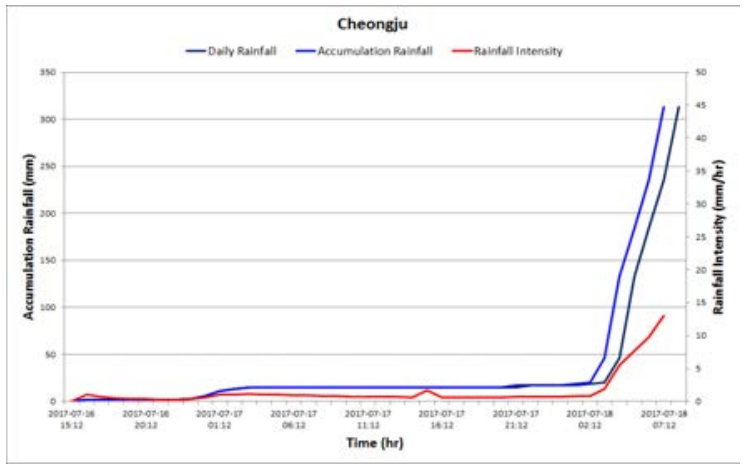

Figure 10: Debris flow forecasting using Korea forest service forecasting criteria (Cheongju). 
Table 4: Estimation of rainfall triggering index (Cheongju).

\begin{tabular}{|l|c|c|c|c|c|c|c|}
\hline $\begin{array}{l}\text { Occurrence } \\
\text { area }\end{array}$ & $\begin{array}{c}\text { Occurrence } \\
\text { time }\end{array}$ & $\begin{array}{c}\text { Duration } \\
\text { of rainfall } \\
(\mathrm{hr})\end{array}$ & $\begin{array}{c}\text { Accumulative } \\
\text { rainfall (mm) }\end{array}$ & $\begin{array}{c}\text { Intensity } \\
(\mathrm{mm} / \mathrm{hr})\end{array}$ & RTI & $\begin{array}{c}\text { RTI } \\
\text { forecasting }\end{array}$ & $\begin{array}{c}\text { Debris flow } \\
\text { forecasting }\end{array}$ \\
\hline \multirow{3}{*}{ Cheongju } & $\begin{array}{c}2017-07-16 \\
15: 12\end{array}$ & 42 & 313 & 7.45 & 2332 & $\begin{array}{c}\text { Emergency } \\
\text { warning }\end{array}$ & Alarm \\
\cline { 2 - 8 } & 3 hours & 39 & 133 & 3.41 & 453 & Advisory & - \\
\cline { 2 - 8 } & 6 hours & 36 & 19 & 0.53 & 10 & Advisory & - \\
\cline { 2 - 8 } & 12 hours & 30 & 15 & 0.5 & 7.5 & Advisory & - \\
\hline
\end{tabular}

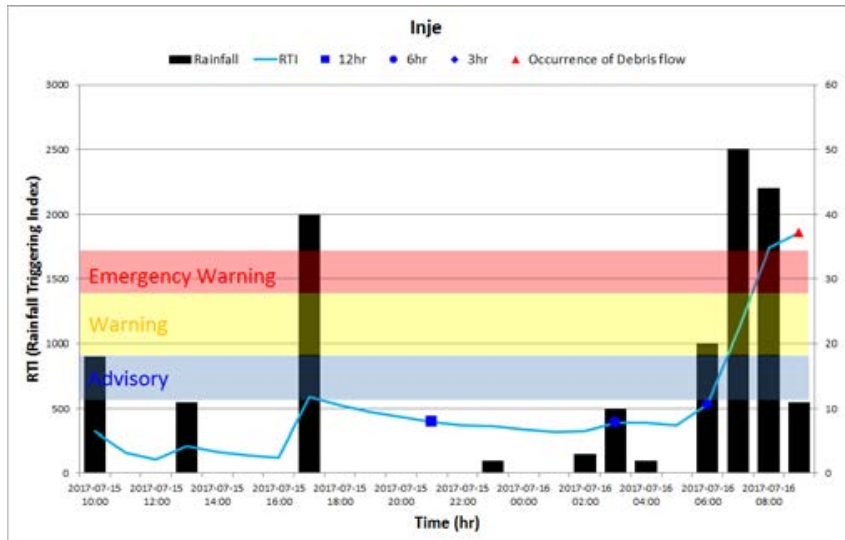

Figure 11: Debris flow forecasting using RTI (Inje).

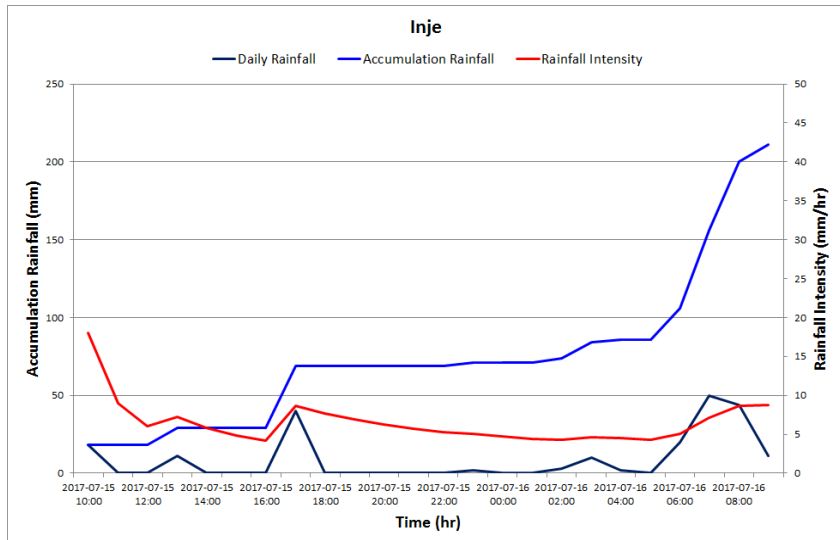

Figure 12: Debris flow forecasting using the Korea forest service forecasting criteria (Inje). 
Table 5: Estimation of rainfall triggering index (Inje).

\begin{tabular}{|l|c|c|c|c|c|c|c|}
\hline \multirow{2}{*}{$\begin{array}{l}\text { Occurrence } \\
\text { area }\end{array}$} & $\begin{array}{c}\text { Occurrence } \\
\text { time }\end{array}$ & $\begin{array}{c}\text { Duration } \\
\text { of rainfall } \\
\text { (hr) }\end{array}$ & $\begin{array}{c}\text { Accumulative } \\
\text { rainfall (mm) }\end{array}$ & $\begin{array}{c}\text { Intensity } \\
(\mathrm{mm} / \mathrm{hr})\end{array}$ & RTI & $\begin{array}{c}\text { RTI } \\
\text { forecasting }\end{array}$ & $\begin{array}{c}\text { Debris flow } \\
\text { forecasting }\end{array}$ \\
\hline \multirow{2}{*}{ Inje } & $\begin{array}{c}2017-07-15 \\
10: 00\end{array}$ & 24 & 211 & 8.8 & 1855 & $\begin{array}{c}\text { Emergency } \\
\text { warning }\end{array}$ & Warning \\
\cline { 2 - 8 } & 3 hours & 21 & 106 & 5 & 535 & Advisory & - \\
\cline { 2 - 8 } & 6 hours & 18 & 84 & 4.6 & 392 & Advisory & - \\
\cline { 2 - 8 } & 12 hours & 12 & 69 & 5.8 & 396 & Advisory & - \\
\hline
\end{tabular}

\section{CONCLUSION}

In this study, the rainfall data of 50 spots affected by debris flows from 2012 to 2013 were analyzed to estimate the RTI. When rainfall lasted for at least 24 consecutive hours within a total rainfall period of up to 7 days right before the debris flow a direct factor that could trigger the event it was considered preceding rainfall. However, when it lasted for less than 24 consecutive hours, it was excluded from the estimation. The estimated RTI was divided into three levels: advisory (10-70\%), warning (70-90\%), and emergency warning (over $90 \%$ ). To review the feasibility of the RTI based on the debris flow caution and warning criteria provided by the KFS, the estimated RTI were applied to previous debris flows that caused serious damage in Umyeonsan Mountain, Seoul, Cheongju-si, and Inje-gun to identify the maximum response period by estimating the levels at 3, 6, and 12 hours before the debris flows occurred. The results are thus described below. For the case of Umyeonsan, the RTI was at the "emergency warning" level with over $90 \%$ of the debris flow risk. Similarly, the KFS debris flow criteria was at the "warning" level. For the cases of Cheongju and Inje, the RTI was at the "emergency warning" level with over $90 \%$ of the debris flow risk. On the other hand, the debris flow criteria were at the "warning" and "caution" for the cases of Cheongju and Inje, respectively.

For the response time, it was found that the RTI was at the "warning" level with a 70\% risk at 3 hours before the occurrence; "advisory" with 10\% risk at 6 hours before; and less than $10 \%$ risk at 12 hours before. These results indicate that response to the disaster could have been provided three hours before it occurred. For the case of Cheongju, the RTI was less than $10 \%$ at 3,6 , and 12 hours prior to the occurrence, suggesting that the short, intense, 5-hour rainfall caused the debris flow. Similarly, for the Inje case, the RTI was less than $10 \%$ at 3,6 , and 12 hours prior to the event, which also means that the disaster was due to the short, intense rainfall. In view of these results, it can be concluded that there is a need to establish more specific and detailed forecasting and warning criteria for debris flows.

\section{ACKNOWLEDGEMENT}

This research was supported by a grant (MOIS-DP-2015-05) through the Disaster and Safety Management Institute funded by Ministry of the Interior and Safety of Korean government.

\section{REFERENCES}

[1] Korea Meteorological Administration, Korean peninsula climate changes forecast report, 1-3, 2016. 
[2] Nam, D.H., Lee, S.H., Jun, K.W. \& Kim, B.S., Study on the debris flow movement and the run-out calculation using the coupling of flood runoff model and debris flow model. Crisisonomy, 12(8), pp. 131-143, 2016.

[3] Gangwon Development Research Institute, Characteristics and Protective Measures against Natural Hazards in the Mountain Areas of Gangwon Province, pp. 1-77, 2008.

[4] Yune, C.Y., Jun, K.J., Kim, K.S., Kim, G.H. \& Lee, S.W., Analysis of slope hazardtriggering rainfall characteristics in Gangwon province by database construction. Korea Geotechnical Society, 26(10), pp. 27-38, 2010.

[5] Jan, C.D. \& Lee, M.H., A debris-flow rainfall-based warning model. Journal of Chinese Soil and Water Conservation, 35(3), pp. 275-285, 2004.

[6] Korea Forest Service, Landslide forecast criteria, 2012. Online. http://sansatai.forest.go.kr.

[7] Jeong, J.W., Development on early warning criteria for debris-flow using real time rainfall monitoring. MSc thesis, Seokyeong University, pp. 1-117, 2015.

[8] Yu, B.I., Study on flash floods and debris flow guidance of Gangwon-do, Kangwon National University, pp. 1-77, 2015. 